\title{
Etelcalcetide: un nuovo calciomimetico per la terapia dell'iperparatiroidismo secondario nei pazienti in emodialisi
}

\author{
Monica Limardo, Giuseppe Pontoriero \\ S.C. Nefrologia e Dialisi ASST Lecco, Ospedale A. Manzoni, Lecco
}

\begin{abstract}
Etelcalcetide: a new intravenous calcimimetic for the treatment of secondary hyperparathyroidism in haemodialysis patients

Etelcalcetide hydrochloride (also known as AMG 416) is a new, second generation, long-acting calcimimetic agent that is administered intravenously. In November 2016 it received its first approval in the UE for the treatment of secondary hyperparathyroidism (SHPT) in adult haemodialysis patients.

This article summarizes the results of pre-clinical and clinical studies which demonstrated that Etelcalcetide decreases effectively PTH levels. Although questions about this new compound remain open, and further studies are required to assess clinical hard outcomes as well as long term efficacy and safety, Etelcalcetide represents a new therapeutic option for the challenging treatment of chronic kidney disease-mineral and bone disorder (CKD-MBD).
\end{abstract}

Keywords: AMG 416, Calcimimetic, Chronic kidney disease, Dialysis, Etelcalcetide, Secondary Hyperparathyroidism

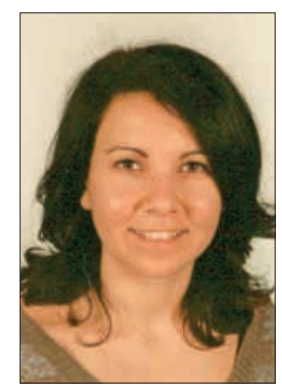

Monica Limardo
Etelcalcetide, anche noto come AMG 416, è il calciomimetico di seconda generazione recentemente autorizzato dall'EMA (European Medicines Agency) (1) per la terapia dell'iperparatiroidismo secondario (SHPT) nei pazienti in emodialisi cronica; la FDA (Food and Drug Administration) lo scorso agosto aveva rilasciato una complete response letter (CRL) nella quale comunicava che non intendeva al momento rilasciarne

\section{l'approvazione $(2,3)$.}

Etelcalcetide è un octapeptide contenente quattro residui di D-arginina, che agisce come attivatore allosterico dei recettori del calcio (4). Questi recettori sono espressi nelle

Accepted: January 14, 2017

Published online: February 2, 2017

Indirizzo per la corrispondenza:

Dr.ssa Monica Limardo

Struttura Complessa di Nefrologia e Dialisi

ASST Lecco

Via Dell'Eremo 9/11

23900 Lecco

m.limardo@asst-lecco.it cellule paratiroidee e negli altri tessuti (renale, scheletrico e nelle cellule parafollicolari della tiroide) coinvolti nell'omeostasi sistemica del Ca++. Come modulatori allosterici, i calciomimetici aumentano la sensibilità dei recettori del calcio all'attivazione del $\mathrm{Ca}++$ extracellulare.

Studi preclinici hanno dimostrato che la somministrazione in singolo bolo di Etelcalcetide induce una rapida riduzione dei livelli di ormone paratiroideo (PTH) in ratti con insufficienza renale acuta (5), mentre la somministrazione ripetuta in ratti sottoposti a nefrectomia $5 / 6$, oltre ad abbassare significativamente i livelli di PTH, porta a una riduzione delle calcificazioni vascolari e dell'iperplasia iperparatiroidea (6). Nei volontari sani, Etelcalcetide riduce in modo rapido, proporzionale al dosaggio e sostenuto, i livelli di PTH, calcio ionizzato e fibroblast growth factor 23 (FGF23) (7).

A differenza del primo calciomimetico, Cinacalcet, disponibile nella formulazione orale, Etelcalcetide viene somministrato per via endovenosa al termine della seduta emodialitica.

L'introduzione del primo calciomimetico disponibile, Cinacalcet (8), ha modificato negli ultimi anni la terapia dell'iperparatiroidismo secondario per i pazienti in dialisi, consentendo di disporre di un farmaco che riduce efficacemente i livelli di PTH, senza aumentare i livelli ematici di calcio e fosforo. Tuttavia, nella pratica clinica, l'impiego di Cinacalcet può essere limitato dai possibili effetti collaterali gastrointestinali e quindi dalla ridotta compliance terapeutica (9). Etelcalcetide è stato 
sviluppato con l'obiettivo di migliorare la tollerabilità gastrointestinale, la compliance al trattamento e conseguentemente l'efficacia della terapia calciomimetica. Infatti, è stato ipotizzato che la somministrazione endovenosa non esponga il lume gastrointestinale a una concentrazione significativa di calciomimetico come nel caso di Cinacalcet (7); inoltre, in studi sulla distribuzione del farmaco, Etelcalcetide non sembra superare in maniera significativa la barriera emato-encefalica e ciò potrebbe mitigare i rischi di eventuali effetti gastrointestinali mediati dal sistema nervoso centrale (6). Un beneficio aggiuntivo per i pazienti potrebbe essere trovato nella riduzione del numero di compresse da assumere al domicilio.

Etelcalcetide e Cinacalcet si differenziano, oltre che per la struttura, anche per il profilo farmacocinetico nei pazienti con malattia renale cronica (CKD): il profilo farmacocinetico di Cinacalcet non è condizionato dal grado di compromissione renale (tuttavia si segnala che gli alimenti possono interferire con la sua biodisponibilità); Etelcalcetide, invece, essendo eliminato quasi esclusivamente per via renale o attraverso la dialisi (10), persiste in circolo nei pazienti con funzione renale residua assai ridotta o assente (11). Ne consegue una maggiore durata d'azione: una singola somministrazione endovenosa di Etelcalcetide può ridurre i livelli di PTH fino a 72 ore nei pazienti in emodialisi (12).

Due studi clinici di fase III randomizzati in doppio cieco, controllati versus placebo, della durata di 6 mesi, sono stati condotti da Block et al $(13,14)$ in un totale di 1023 pazienti con iperparatiroidismo secondario (SHPT) (PTH $>400 \mathrm{pg} / \mathrm{mL}$ nonostante le terapie in atto) in emodialisi trisettimanale. In entrambi questi studi, Etelcalcetide oppure placebo venivano somministrati e.v. al termine di ciascuna seduta dialitica, titolandone il dosaggio ogni 4 settimane fino a una dose massima di $15 \mathrm{mg} 3$ volte a settimana, per raggiungere il livello target di PTH $\leq 300 \mathrm{pg} / \mathrm{mL}$. I pazienti sono stati mantenuti con una concentrazione stabile di calcio nel dialisato $\geq 2.25 \mathrm{mEq} / \mathrm{L}$. L'end-point primario, rappresentato dalla percentuale di pazienti con una riduzione $>30 \%$ dei livelli di PTH rispetto al basale, è stato raggiunto nel $74.7 \%$ dei pazienti trattati con Etelcalcetide rispetto all' $8.9 \%$ dei trattati con placebo. Tra gli end-point secondari, una proporzione significativamente maggiore di pazienti $(51.5 \%$ nei pazienti in trattamento con Etelcalcetide versus $5.9 \%$ dei pazienti in placebo) raggiunse livelli di $\mathrm{PTH}<300 \mathrm{pg} / \mathrm{mL}$, partendo da valori basali di PTH sovrapponibili (superiori a 800 pg/mL in entrambi gli studi).

Nei pazienti trattati con Etelcalcetide vi era inoltre una riduzione significativa della fosforemia rispetto ai pazienti in placebo $(-7.71 \%$ versus $-1.31 \%$ nel primo studio e $-9.63 \%$ versus $-1.6 \%$ nel secondo studio), oltre che dei livelli sierici di calcio corretto per l'albumina (-7.29\% versus $1.18 \%$ nel primo studio e $-6.69 \%$ versus $0.58 \%$ nel secondo studio). Di rilievo, nei pazienti in trattamento con Etelcalcetide, si osservò un maggior numero di episodi di ipocalcemia sintomatica (7\% versus $0.2 \%$ ). Per quanto concerne gli altri eventi avversi, spasmi muscolari e sintomi gastrointestinali (nausea, vomito e diarrea), vennero osservati con maggiore frequenza nei pazienti trattati con Etelcalcetide. I tassi di mortalità e la frequenza di episodi convulsivi risultarono simili nei due bracci di trattamento $(13,14)$.

Si segnala inoltre una riduzione significativa dei livelli di FGF23 nei due terzi dei pazienti trattati con Etelcalcetide, rispetto al $30 \%$ dei pazienti in terapia con placebo $(13,14)$.

Risultati simili di riduzione dei livelli di PTH, calcio, fosforo e FGF23 sono stati confermati anche in uno studio randomizzato, controllato, in doppio cieco di fase III, condotto in Giappone (15), nel quale l'end-point primario era rappresentato dal raggiungimento di un target inferiore di PTH $(60-240 \mathrm{pg} / \mathrm{mL})$, secondo quanto raccomandato dalle Linee Guida nipponiche.

In uno studio di fase 3 condotto su 120 pazienti emodializzati (16) è stato evidenziato che è possibile effettuare in maniera sicura uno switch di terapia da Cinacalcet a Etelcalcetide $5 \mathrm{mg}$ 3 volte a settimana, dopo un wash-out di almeno 7 giorni da Cinacalcet e a condizione che il calcio corretto sia $\geq 8.3 \mathrm{mg} / \mathrm{dL}$. Questo switch è stato effettuato senza variazioni significative nella terapia con vitamina $D$ e supplementi o chelanti a base di calcio. Di rilievo, i pazienti che nel periodo precedente assumevano Cinacalcet al dosaggio di $30 \mathrm{mg}$ presentarono, rispetto ai pazienti precedentemente trattati con 60 o 90 mg, i livelli più bassi di calcemia sia dopo il wash-out da Cinacalcet sia nel corso del trattamento con Etelcalcetide (16).

In uno studio randomizzato in doppio cieco, controllato, della durata di sei mesi, Block et al (17) hanno messo a confronto l'efficacia e la sicurezza di Etelcalcetide con quelle di Cinacalcet in 683 pazienti in emodialisi affetti da SHPT (PTH $>500 \mathrm{pg} / \mathrm{mL}$ nonostante le terapie in atto). In questo studio è stato raggiunto l'end-point primario di non-inferiorità, misurato come la proporzione di pazienti con una riduzione del PTH $>30 \%$ rispetto al basale. Inoltre Etelcalcetide è risultato superiore rispetto a Cinacalcet nel raggiungimento degli endpoint secondari, rappresentati dalla proporzione di pazienti con una riduzione del PTH rispetto al basale $>30 \%$ (68.2\% versus $57.7 \% p=0.004$ ) e superiore al $50 \%$ (52.4\% versus $40.2 \%$ $p=0.001)$.

Non sono state invece osservate differenze significative tra i due gruppi per quanto riguarda l'end-point secondario relativo alla valutazione del numero medio di giorni di vomito o nausea alla settimana nelle prime 8 settimane. La percentuale di eventi avversi è risultata sovrapponibile per i due trattamenti. Gli eventi avversi più comuni sono stati: riduzione dei livelli di calcemia (68.9\% Etelcalcetide versus 59.8\% Cinacalcet), nausea (18.3\% Etelcalcetide versus 22.6\% Cinacalcet), vomito (13.3\% Etelcalcetide verso $13.8 \%$ Cinacalcet) e diarrea (6.2\% Etelcalcetide verso $10.3 \%$ Cinacalcet). Gli episodi di ipocalcemia sintomatica sono stati riportati nel $5 \%$ dei pazienti in terapia con Etelcalcetide rispetto al $2.3 \%$ dei pazienti trattati con Cinacalcet.

È stato osservato uno sbilanciamento nel numero di eventi avversi correlati a scompenso cardiaco nel $3 \%$ dei pazienti trattati con Etelcalcetide rispetto allo $0.6 \%$ dei pazienti trattati 
con Cinacalcet. Nessuno degli eventi fatali o seri di scompenso cardiaco è avvenuto in corso di ipocalcemia. Le percentuali di eventi avversi seri e fatali sono risultate simili nei pazienti trattati con Etelcalcetide e nei pazienti trattati con Cinacalcet (17).

Negli studi di Block et al $(13,17)$, la titolazione del farmaco è stata effettuata in modo centralizzato in relazione ai valori di PTH e calcio e i valori di PTH non erano noti ai medici che seguivano clinicamente i pazienti. Per motivi di sicurezza, i valori di calcemia erano invece noti e i medici potevano modificare la restante terapia del SHPT in relazione a eventuali variazioni. I pazienti trattati con Etelcalcetide in questi studi sono risultati più soggetti a ricevere terapie a base di calcio (supplementi o chelanti) e vitamina D e ad avere incrementi nella concentrazione di calcio nel dialisato. Malgrado ciò, i pazienti trattati con Etelcalcetide presentavano più bassi valori di calcio e più frequentemente spasmi muscolari e prolungamento dell'intervallo QT rispetto ai pazienti in terapia con placebo (18).

Uno studio open-label della durata di 52 settimane (di estensione di due precedenti studi in doppio cieco) è stato condotto su 891 pazienti in emodialisi affetti da SHPT per caratterizzare la sicurezza e l'efficacia di Etelcalcetide (19). Tutti i soggetti hanno ricevuto Etelcalcetide, che veniva titolato da una dose iniziale di $5 \mathrm{mg}$ fino a una dose massima di $15 \mathrm{mg}$ per raggiungere livelli target di PTH $\leq 300 \mathrm{pg} / \mathrm{mL}$. In questo studio sono stati confermati i risultati degli studi precedenti, sia per quanto riguarda il mantenimento dell'efficacia della terapia nel lungo termine sia per quanto riguarda i risultati relativi alla sicurezza (19).

Un ulteriore studio open-label di fase III (NCT02102204) della durata di circa 2 anni e mezzo è ancora in corso e dovrebbe concludersi nel Giugno del 2017. Questo studio ha arruolato 902 pazienti che avevano partecipato a precedenti studi con Etelcalcetide, con l'obiettivo di valutarne la sicurezza e la tollerabilità sul lungo termine (20).

Sulla base dei risultati disponibili, Etelcalcetide sembra aver una pari o persino maggiore efficacia in termini di riduzione del PTH rispetto a Cinacalcet, tuttavia rispetto all'atteso non presenta vantaggi in termini di tollerabilità gastrointestinale e frequenza di eventi avversi rispetto al calciomimetico orale. Per quanto riguarda la tollerabilità gastrointestinale, è verosimile che gli effetti indotti da entrambi siano di natura sistemica e mediati dall'attivazione di recettori non-paratiroidei, piuttosto che non legati alla concentrazione locale del calciomimetico nel lume gastrointestinale, come precedentemente ipotizzato (18).

Emerge chiaro un rischio significativo di ipocalcemia sintomatica. È stato ipotizzato che l'effetto ipocalcemizzante di Etelcalcetide sia più pronunciato all'inizio della terapia, quando il PTH è più elevato e l'osso è maggiormente demineralizzato, similmente a come avviene nell'ipocalcemia successiva all'intervento chirurgico di paratiroidectomia nei pazienti con CKD (18). È possibile inoltre che l'ipocalcemia sia meno pronunciata nel caso in cui i pazienti inizino la terapia con Etelcalcetide più precocemente rispetto a come è avvenuto in questi studi, che hanno arruolato pazienti in dialisi da lungo tempo e con SHPT non controllato nonostante le terapie in atto (18). Queste ipotesi dovranno essere verificate in futuro. Inoltre, sarebbe interessante avere uno studio rigoroso che valuti se Etelcalcetide, consentendo un miglior controllo del SHPT, è in grado di ridurre la mortalità e gli eventi cardiovascolari maggiori nei pazienti in emodialisi.

Comunque, i livelli di calcio sierico vanno attentamente monitorati nei pazienti in corso di terapia con Etelcalcetide e i pazienti vanno accuratamente informati sul rischio di ipocalcemia e dei sintomi con cui potrebbe manifestarsi.

Inoltre, è importante valutare l'anamnesi patologica e familiare dei pazienti candidati a questa terapia, per individuare la possibile presenza di un'eventuale sindrome congenita del QT lungo o altre condizioni che possano predisporre al prolungamento del QT e all'aritmia ventricolare, oltre alla presenza di un'eventuale funzionalità miocardica ridotta (21).

Con questi accorgimenti, Etelcalcetide rappresenta una nuova strategia terapeutica per il Nefrologo nella gestione spesso complessa dell'iperparatiroidismo secondario nei pazienti emodializzati, con il vantaggio di poter gestire direttamente la terapia al termine della seduta emodialitica, consentendo di migliorare la compliance oltre a ridurre il numero di compresse che il paziente deve assumere a domicilio.

\section{Disclosures}

Financial support: No financial support was received for this submission.

Conflict of interest: The authors have no conflict of interest.

\section{Bibliografia}

1. http://www.ema.europa.eu/ema/index.jsp?curl=pages/ medicines/human/medicines/003995/human_med_002037. jsp\&mid=WC0b01ac058001d124. Accessed January 30, 2017.

2. http://www.amgen.com/media/news-releases/2016/08/amgen-provides-update-on-status-of-parsabiv-etelcalcetide-newdrug-application-nda-submitted-to-the-us-food-and-drug-administration-fda/ Accessed January 30, 2017.

3. Blair HA. Etelcalcetide: first global approval. Drugs. 2016:76(18):1787-1792.

4. Alexander ST, Hunter T, Walter S, et al. Critical Cysteine residues in both the calcium-sensing receptor and the allosteric activator AMG 416 underlie the mechanism of action. Mol Pharmacol. 2015:88:853-865.

5. Walter S, Baruch A, Alexander ST, et al. Comparison of AMG 416 and Cinacalcet in rodent models of uremia. BMC Nephrol. 2014;15(1):81.

6. Walter S, Baruch A., Dong J, et al. Pharmacology of AMG 416 (Velcalcetide), a novel peptide agonist of the calcium-sensing receptor, for the treatment of secondary hyperparathyroidism in hemodialysis patients. J Pharmacol Exp Ther. 2013;346: 229-240.

7. Martin JM, Bell G, Pickhorn K, et al. Velcalcetide (AMG 416), a novel peptide agonist of calcium-sensing receptor, reduces parathyroid hormon and FGF23 levels in healthy male subjects. Nephrol Dial Transplant. 2014:29;385-392.

8. Block GA, Martin KJ, de Francisco AL, et al. Cinacalcet for sec- 
ondary hyperparathyroidism in patients receiving hemodialysis. N Engl J Med. 2004;350:1516-1525.

9. Gincherman Y, Moloney K, McKee C, Coyne DW. Assessment of adherence to cinacalcet by prescription refill rates in hemodialysis patients. Hemodial Int. 2010;14:68-72.

10. Subramanian R, Zhu X, Hock MB, et al. Phamacokinetics, biotransformation and excretion of $\left[{ }^{14} \mathrm{C}\right]$ Etelcalcetide (AMG 416) following a single microtracer intravenous dose in patients with chronic kidney disease on hemodialysis. Clin Pharmacokinet. 2017;56(2):179-192.

11. Nemeth EF, Goodman WG. Calcimimetic and calcilytic drugs: feats, flops and futures. Calcif Tissue Int. 2016; 98: 341-358.

12. Martin KJ, Pickthorn K, Huang S, et al. AMG 416 (velcalcetide) is a novel peptide for the treatment of secondary hyperparathyroidism in a single-dose study in hemodialysis patients. Kidney Int. 2013; 85: 191-197.

13. Block GA, Bushinsky DA, Cunningham J, et al. Effect of etelcalcitide vs placebo on serum parathyroid hormone in patients receiving hemodialysis with secondary hyperparathyroidism: two randomized clinical trials. JAMA. 2017; 317 (2): 146-155.

14. http://www.amgen.com/media/news-releases/2015/05/amgen-presents-pooled-phase-3-amg-416-data-for-the-treatment-of-secondary-hyperparathyroidism-in-patients-withchronic-kidney-disease/. Accessed January 30, 2017.

15. Fukagawa M, Yokoyama K, Shigematsu T, et al. A phase 3, multicentre, randomized, double-blind, placebo controlled parallel-group study to evaluate the efficacy and safety of etelcalcetide (ONO-5163/ AMG 416), a novel intravenous calcimimetic, for secondary hyperparathyroidism in Japanese haemodialsis patients. Nephrol Dial Transplant. 2017. Jan 5; [Epub ahead of print].

16. Liss K, Block GA, Chertow GM, Dehmel B, Sun Y, Spiegel DM. Initiation of AMG 416 After Discontinuation of Cinacalcet. ASN Annual Meeting; November 3-8 2015; San Diego. Abstract TH-PO516. J Am Soc Nephrol. 2015;26: (Abstract Supplement).

17. Block GA, Bushinsky DA, Cheng $S$, et al. Effect of etelcalcetide vs cinacalcet on serum parathyroid hormone in patients receiving hemodialysis with secondary hyperparathyroidism: a randomized clinical trial. JAMA. 2017; 317 (2): 156-164.

18. Middleton JP, Wolf M. Second Chances to Improve ESRD Outcomes With a Second-Generation Calcimimetic. JAMA. 2017; 317 (2): 139-141.

19. Bushinsky DA, Block GA, Cheng $S$, et al. One year efficacy and safety of intravenous (IV) etelcalcetide (AMG 416) in patients on hemodialysis (HD) with secondary hyperparathyroidism (SHPT). Abstract SO030 at $53^{\text {rd }}$ Congress ERA-EDTA; May 21-24 2016; Vienna, Austria. Nephrol Dial Transplant. 2016; 31(Suppl. 1); i13-14.

20. https://clinicaltrials.gov/ct2/show/NCT02102204?term=NCTO 2102204\&rank=1. Accessed January 30, 2017.

21. https://ec.europa.eu/health/documents/community-register/2016/20161111136254/anx_136254_it.pdf. Accessed January 30, 2017. 